\title{
Detection of Channel Variations to Improve Channel Estimation Methods
}

\author{
Adriana Dapena • José A. García-Naya • \\ Paula M. Castro • Vicente Zarzoso
}

Received: date / Accepted: date

\begin{abstract}
In current digital communication systems, channel information is typically acquired by supervised approaches that use pilot symbols included in the transmit frames. Given that pilot symbols do not convey user data, they penalize throughput, spectral efficiency, and transmit energy consumption of the system. Unsupervised channel estimation algorithms could be used to mitigate the aforementioned drawbacks although they present higher computational complexity than that offered by supervised ones.

This paper proposes a simple decision method suitable for slowly-varying channels to determine whether the channel has suffered a significant variation, which requires to estimate the matrix of the recently changed channel. Otherwise, a previous estimate is used to recover the transmitted symbols. The main advantage of this method is that the decision criterion is only based on information acquired during the time frame synchronization, which is carried out at the receiver. We show that the proposed criterion provides a considerable
\end{abstract}

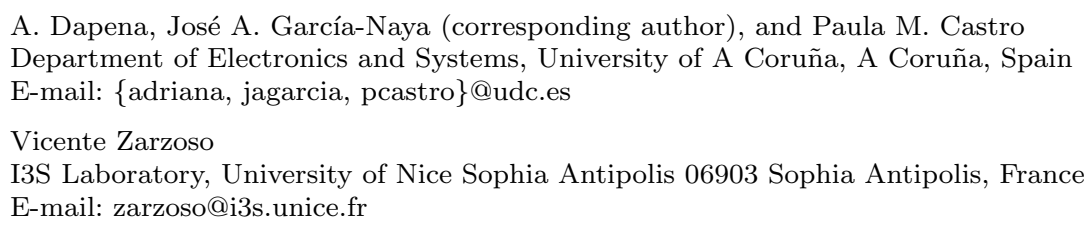


improvement of computational complexity for both supervised and unsupervised methods, without incurring in a penalization in terms of symbol error ratio. Specifically, we consider systems that make use of the popular Alamouti code. Performance evaluation is accomplished by means of simulated channels as well as making use of indoor wireless channels measured using a testbed.

Keywords Channel estimation · Supervised approach · Unsupervised approach $\cdot$ Alamouti code

\section{Introduction}

Supervised channel estimation is the best-known and most widely used method to acquire Channel State Information (CSI) at the receiver side. It requires to transmit pilot symbols which produces a degradation in terms of throughput, spectral efficiency, and transmit energy. The so-called unsupervised techniques are able to estimate the channel coefficients directly from the observations, without requiring pilot symbols. More generally, they assume some properties of the transmitted signal, such as finite alphabet, statistical independence, spectral color, etc. [5] as well as an invertible channel matrix. Unfortunately, unsupervised approaches present a high computational complexity since they generally require to estimate higher-order statistics by sample averaging over all the received symbols.

In previous works $[4,6]$, several methods based on detecting wireless channel variations to reduce the amount of pilot symbols used for channel estimation have been proposed by the authors under scenarios implementing both forward and feedback links. The feedback link was used to send information to the transmitter with the goal of adapting different transmit parameters (e.g. modulation and coding scheme) to the propagation conditions [15]. The method proposed in $[4,6]$ estimated the channel using an unsupervised algo- 
rithm and, subsequently, compared such an estimation to a previous one. When a significant fluctuation was detected, the receiver requested pilots to the transmitter using the limited feedback channel. Since the channel information was estimated per each received frame, the main drawback of those approaches was their computational complexity. Furthermore, the performance analysis shown in $[4,6]$ was restricted to synthetically generated and spatially-white Rayleigh-distributed wireless channels.

The present work proposes a novel method for tracking wireless channel variations taking advantage of the calculations already necessary for time synchronization of acquired frames. Moreover, with this proposal the receiver complexity is not penalized. Basically, the idea consists in making use of correlation-like operations to obtain a rough estimation of the wireless channel. Such correlations are already employed at the receiver for implementing synchronization tasks. Therefore, our proposal takes advantage of such calculations to obtain an inexpensive and approximate channel estimation with the objective of tracking significant channel variations. As it will be shown throughout this work, CSI obtained at the receiver in such an early stage is sufficient to determine if the CSI has changed significantly and hence the channel must be re-estimated. Note that such a CSI acquisition takes place only if the decision criterion determines a significant channel variation, which leads to a considerable reduction of computational complexity for both supervised and unsupervised methods, without penalizing the performance (in terms of Signal to Noise Ratio). Additionally, the proposed method also allows for reducing the overhead due to pilot symbols because the feedback channel can be used to indicate whether to include pilots in the frame to estimate the channel or, on the contrary, the transmitter can send only user symbols. Note, however, that the method proposed in this paper is applicable even in the absence of 
feedback channels but, in this case, pilot symbols are included in every frame, thus loosing part of the potential offered by this proposal.

The aforementioned method is particularized for the specific case of Alamouti Orthogonal Space-Time Block Code (OSTBC) [1] with two transmit antennas and a single receive antenna. Nevertheless, the idea of making use of CSI obtained from synchronization stages at the receiver is more general, enabling its use in a wider range of wireless communication systems. We decide to use the $(2 \times 1)$ Alamouti OSTBC since it is the only OSTBC capable of achieving full spatial rate for complex constellations. Thus, other OSTBC schemes proposed for more than two transmit antennas suffer from a severe spatial rate loss [21]. Additionally and because of these advantages, the Alamouti OSTBC has been included in recent wireless communication standards, e.g. IEEE 802.11n WiFi [13], or IEEE 802.16-2009 WiMAX [14].

This work is organized as follows. Section 2 describes the signal model and reviews both supervised and unsupervised channel estimation methods. Section 3 explains the synchronization method typically used in current digital communication standards and introduces the proposed technique to detect channel variations. Illustrative results by means of computer simulations are presented in Section 4 for the case of simulated channels, and in Section 5 for the case of measured indoor channels. Finally, concluding remarks are stated in Section 6 .

\section{Digital Communication Scheme}

High data-rate communications are not only limited by noise but also by InterSymbol Interference (ISI) due to the memory of time-dispersive wireless communications channels. Such a channel memory is caused by the dispersive channel impulse response due to the different lengths of the propagation paths 
between the transmitter and the receiver antennas (multipath channel), leading to a frequency-selective channel response.

An efficient method to combat the multipath effect is the multicarrier approach, in which a set of subcarriers are employed to transmit the information in parallel over the channel. A multicarrier system can be implemented efficiently in discrete time using the Inverse Fast Fourier Transform (IFFT) at the transmitter side and the Fast Fourier Transform (FFT) at the receiver side [20]. The main advantage of Orthogonal Frequency-Division Multiplexing (OFDM) when using the so-called cyclic prefix is the transformation of a wideband frequency-selective channel in multiple narrowband frequency-flat subchannels, one for each subcarrier, thus effectively combating the effects of the multipath propagation. Specifically, for a system with two transmitter antennas an only one receiver antena, the signal at the $l$-th subcarrier has the form

$$
x^{(l)}=h_{1}^{(l)} s_{1}^{(l)}+h_{2}^{(l)} s_{2}^{(l)}+n^{(l)},
$$

where $s_{1}^{(l)}$ and $s_{2}^{(l)}, h_{1}^{(l)}$ and $h_{2}^{(l)}$, and $n^{(l)}$ are, respectively, the signals, the channel coefficients and the noise component at the $l$-th subcarrier.

Current standards based on OFDM, e.g. IEEE802.11n and IEEE802.16 $[13,14]$, include the Alamouti OSTBC to decouple the signals transmitted from different antennas. Due to the importance of Alamouti OSTBC, in this paper we will focus our attention on reducing the computational load associated to the channel estimation when coherent detection is used at the receiver. Even though the ideas presented in this paper can also be employed in OFDM schemes, we restrict our analysis to single-carrier ones. From now on, we will remove the references to subcarriers (i.e., the superscript ${ }^{(l)}$ will be removed in all equations). 
2.1 Alamouti Coding Scheme and Channel Estimation

We consider a single-user communication system based on Alamouti OSTBC with two antennas at the transmitter and a single one at the receiver (see Fig. 1). The source symbols $s(t)$ have zero-mean with variance $\sigma_{s}^{2}$, with $t=$ $0,1, \ldots$ representing the discrete time index (symbol number). The signal $x(t)$ observed at the receive antenna is corrupted by additive noise $n(t)$. To simplify the mathematical notations, we define

$$
s_{1}(k)=s(2 k), \quad s_{2}(k)=s(2 k+1), \quad k=0,1,2, \ldots
$$

and analogous expressions for $x_{1}(k), x_{2}(k), n_{1}(k)$ and $n_{2}(k)$. We make the following assumptions on the transmitted signals, the noise, and the channel:

A1 The transmit signals, $s_{1}(k)$ and $s_{2}(k)$, are complex-valued, zero-mean, stationary, non Gaussian-distributed and statistically independent; whereas their exact probability density function (pdf) is unknown.

A2 The noise, $n_{1}(k)$ and $n_{2}(k)$, is a zero-mean white Gaussian process with unknown variance $\sigma_{n}^{2}$.

A3 The channel coefficients, $h_{1}$ and $h_{2}$, are constant over the observation window (slowly-varying flat-fading channel), but otherwise unknown.

The $(2 \times 1)$ Alamouti coding scheme utilizes the following coding matrix:

$$
\mathbf{S}_{k}=\left[\begin{array}{cc}
s_{1}(k) & s_{2}(k) \\
-s_{2}(k)^{*} & s_{1}(k)^{*}
\end{array}\right]
$$

This means that, in odd time instants $s_{1}(k)$ is transmitted by the first antenna while $s_{2}(k)$ is sent by the second antenna. In even time instants, $s_{1}(k)^{*}$ is transmitted by the second antenna and $-s_{2}(k)^{*}$ by the first one. Defining the received vector $\mathbf{x}_{k}=\left[x_{1}(k) x_{2}(k)\right]^{\mathrm{T}}$, the channel vector $\mathbf{h}=\left[h_{1} h_{2}\right]^{\mathrm{T}}$ and 
the noise vector $\mathbf{n}_{k}=\left[n_{1}(k) n_{2}(k)\right]^{\mathrm{T}}$, we can write the following signal model

$$
\mathbf{x}_{k}=\mathbf{S}_{k} \mathbf{h}+\mathbf{n}_{k}
$$

Under assumptions A1 to A3 in Appendix A, we show that the channel coefficients can be expressed as follows:

$$
\begin{aligned}
& \hat{h}_{1, \mathrm{ML}}=\frac{1}{2 N \hat{\sigma}_{s}^{2}} \sum_{k=0}^{N-1}\left(s_{1}(k)^{*} x_{1}(k)-s_{2}(k) x_{2}(k)\right) \\
& \hat{h}_{2, \mathrm{ML}}=\frac{1}{2 N \hat{\sigma}_{s}^{2}} \sum_{k=0}^{N-1}\left(s_{2}(k)^{*} x_{1}(k)+s_{1}(k) x_{2}(k)\right) .
\end{aligned}
$$

Hence, the ML estimates are essentially determined by cross-correlations between the received signals and the transmitted symbols. In practice, the transmitted signals are known during a finite time interval (pilot symbols).

Transmission of pilot symbols can be avoided taking advantage of unsupervised approaches. They can be classified according to the statistical information needed to perform the channel estimation in Second-Order Statistics (SOS) and Higher-Order Statistics (HOS) based approaches.

The limitations of using SOS in Alamouti coding scheme were first focused on by Shahbazpanahi et al. in [19] and, more recently, by other authors $[18,22]$. On the other hand, a family of HOS algorithms has been proposed in the context of Blind Source Separation (BSS) considering the diagonalization of fourth-order cross-cumulant matrices. In particular, Cardoso et al. [3] have proposed the popular Joint Approximate Diagonalization of Eigenmatrices (JADE) algorithm in which the channel matrix is obtained by jointly diagonalizing a set of fourth-order cross-cumulant matrices. The orthogonality property of the channel matrix used in the Alamouti coding scheme has been employed in $[2,7]$ to reduce the computational complexity of JADE. In this 
paper, we will consider the technique proposed by Dapena et al. [7] termed Blind Channel Estimation based on Eigenvalue Spread (BCEES), which selects the fourth-order cross-cumulant matrix to be diagonalized taking into account the absolute difference between the eigenvalues (which is referred to as eigenvalue spread). This method provides similar performance than JADE with a considerable reduction on computational load.

\section{Synchronization and Detection of Channel Variations}

In real-world transmissions, several operations must be performed before channel estimation. Current standards define preamble sequences ${ }^{1}$ to correct phase shift, to estimate signal power, to perform time and frequency synchronization, etc. [17]. Note that it is not needed to code the preambles using Alamouti OSTBC because we can impose some restrictions to the sequence to transmit.

Since we have only a single antenna, after doing the FFT at the receiver, the receive signal at each subcarrier has the form (see Eq. (1))

$$
x(k)=h_{1} p_{1}(k)+h_{2} p_{2}(k)+n(k), \quad k=0,1,2, \ldots,
$$

where $p_{1}(k)$ and $p_{2}(k)$ represent the preambles transmitted by the first and the second antenna, respectively. We assume that the channel remain constant during the transmission of the preambles.

The performance of our method does not depend on the specific sequence, but it is needed to guarantee that $p_{1}(k)$ and $p_{2}(k)$ are orthogonal, i.e.,

$$
\sum_{k=1}^{P} p_{1}(k) p_{2}(k)^{*}=0
$$

1 Actually, current wireless communication standards define other parts of the frames for control data which are not considered in this work. 
From the existing methods to select the preamble structure, we have selected the proposed in [16] which consists in defining the preamble sequence of $P$ symbols by repeating $L$ times a sub-sequence of $P / L$ symbols. The periodicity nature of the preamble equips timing synchronization with robustness against frequency offsets.

The synchronization procedure for the $i$-th frame, computes the crosscorrelation of the observed signal in Eq. (6) with the preambles $p_{1}(k)$ and $p_{2}(k)$. From Eq. (6), we obtain the following expressions

$$
\begin{aligned}
\sum_{k=1}^{P}\left[x(k) p_{1}(k)^{*}\right] & =\sum_{k=1}^{P}\left[h_{1}\left|p_{1}(k)\right|^{2}+h_{2} p_{2}(k) p_{1}(k)^{*}+n(k) p_{1}(k)^{*}\right] \\
& =\sum_{k=1}^{P}\left[h_{1}\left|p_{1}(k)\right|^{2}\right]+\sum_{k=1}^{P}\left[h_{2} p_{2}(k) p_{1}(k)^{*}\right]+\sum_{k=1}^{P}\left[n(k) p_{1}(k)^{*}\right]
\end{aligned}
$$

Since the preambles are orthogonal and assuming that the pramble length is enough to compensate for the amount of received noise, the above expression takes the form

$$
\sum_{k=1}^{P}\left[x(k) p_{1}(k)^{*}\right]=h_{1} \sum_{k=1}^{P}\left|p_{1}(k)\right|^{2}
$$

Using a similar reasoning, we have

$$
\sum_{k=1}^{P}\left[x(k) p_{2}(k)^{*}\right]=h_{2} \sum_{k=1}^{P}\left|p_{2}(k)\right|^{2}
$$

As a consequence, the synchronization procedure obtains rough estimations of the channel coefficients $h_{1}$ and $h_{2}$ as follows

$$
\hat{h}_{1}=\frac{\sum_{k=1}^{P}\left[x(k) p_{1}(k)^{*}\right]}{\sum_{k=1}^{P}\left[\left|p_{1}(k)\right|^{2}\right]}, \quad \hat{h}_{2}=\frac{\sum_{k=1}^{P}\left[x(k) p_{2}(k)^{*}\right]}{\sum_{k=1}^{P}\left[\left|p_{2}(k)\right|^{2}\right]}
$$

We propose to use these estimates to determine the time instants when the channel suffers a significant variation and, therefore, the time instants 
when channel matrix must be estimated. For this aim, we compare the values corresponding to two consecutive frames, denoted by the indices $i$ and $i-1$, as shown the following procedure:

- Step 1. Compute the differences:

Difference $_{1}[i]=\left|\hat{h}_{1}[i]-\hat{h}_{1}[i-1]\right|$,

Difference $_{2}[i]=\left|\hat{h}_{2}[i]-\hat{h}_{2}[i-1]\right|$.

- Step 2. Decide when the channel has significantly changed using the following decision criterion in which $\alpha$ is a positive real-valued threshold:

If $\left(\right.$ Difference $\left._{1}[i]>\alpha\right)$ OR ( Difference $\left._{2}[i]>\alpha\right) \rightarrow$ channel estimation.

The inclusion of this decision rule allows us to reduce the computational complexity as well as the average power consumption of the estimation algorithm (in terms of the energy wasted in processing symbols at the receiver side) since the channel matrix is estimated only when a significant variation is detected. During the rest of the time, a previous estimate is used to recover the transmitted symbols.

Current wireless communication standards make use of feedback channels connecting the receiver and the transmitter sides of the link to periodically send CSI back to the transmitter. In Frequency Division Duplex (FDD) systems, this feedback channel is limited in terms of throughput but it can be used to send a "flag" indicating if the channel has suffered a significant variation which requires the transmission of pilot symbols. For these systems, we can define two frame types:

- Classical frames, containing both pilot and user data symbols, and

- User frames, containing only user data symbols. 
Note that both frame types include a preamble for time synchronization as well as for channel tracking. It is important to emphasize that the design of the frames is solely based on the subsequent performance study. Notice also that including a parameter in the frame indicating whether pilots are included or not in the frame is not of an extraordinary complexity. Moreover, current wireless standards already include such parameters to indicate for example the modulation type, coding rate, etc.

During the frame synchronization procedure, the criterion detailed above is used to determine if a channel variation has occurred. When this happens, the transmitter is notified that a classical frame must be transmitted. Subsequently, the channel is estimated from this classical frame and the estimate is used to recover both current (classical frame) and previous frame (user data frame). Otherwise, when no variations are detected, the channel is equalized by means of the current channel estimate, which has been obtained from the last classical frame transmitted. Notice that we are making use of our assumption of a slowly-varying channel.

From now on, we will refer to Decision-Aided (DA) as the approaches using the proposed decision rule, i.e. DA-Supervised, DA-JADE, and DA-BCEES.

\section{Performance Evaluation Based on Simulated Channels}

We evaluate the performance of the channel estimation approaches studied in this work by considering randomly generated channels with spatially-white Rayleigh-distributed coefficients, while noise terms are AWGN. The experiments have been performed using QPSK source symbols coded with Alamouti OSTBC. A total of 20 frames consisting of 200 symbols per transmit antenna. The channel matrix remains constant during the transmission of 5 frames; hence the 20 frames experience 4 different channel realizations. Finally, the 
results have been averaged over 1000 independent realizations. We stress that the frame structure was solely designed with the purpose of comparing the performance of the evaluated methods.

\subsection{Supervised Approaches}

The first experiment has been carried out to determine the minimum number of pilot symbols - expressed with respect to the total number of symbols per frame - needed by the supervised channel estimation method (see Eq. (5)). The channel matrix is estimated in all frames, without using the proposed decision criterion. We have considered that each frame contains $1 \%, 2 \%, 4 \%$, or $50 \%$ of pilot symbols per antenna (i.e. each frame contains, respectively, 2 , 4,8 , or 100 pilot symbols per antenna, while the remaining symbols correspond to user data). Figure 2 plots Symbol Error Ratio (SER) versus Signal-to-Noise Ratio (SNR) for simulated channels and shows that using $4 \%$ of pilot symbols (8 pilot symbols per frame and per transmit antenna) is enough to achieve a good performance. For this reason, 8 pilot symbols per frame will be used in the rest of the experiments.

Next, we consider the problem that arises from selecting the threshold value $\alpha$ and the preamble size used for the proposed decision criterion. The base sequence transmitted by the first antenna is shown in Table 1, the second antenna transmits a base sequence formed by 1's. In both antennas, the sequence is repeated with the pattern $(-+--)$ proposed in [16].

In order to determine the threshold, we will use a preamble of 80 symbols per antenna. A figure of merit of the difference - in terms of SER - between the DA-Supervised and the supervised approaches is defined as follows

$$
\epsilon_{\mathrm{SER}}=\frac{\mathrm{SER}_{\mathrm{DA} \text {-Supervised }}-\mathrm{SER}_{\text {Supervised }}}{1+\mathrm{SER}_{\mathrm{DA}-\text { Supervised }}} .
$$


The term "1" in the denominator is included to avoid division by zero. Figure 3 shows this difference in SER as well as the percentage of channel utilization (number of channel estimates divided by the total number of frames). It can be seen from the figure that choosing a value of $\alpha=0.4$ the figure of merit defined in Eq. (11) is zero while the algorithm utilization is equal to $25 \%$, which means to estimate the channel only 5 times during the transmission of 20 frames (i.e. for the first frame as well as for each channel variation). From values of $\alpha$ greater than 0.7 the method is not able to detect all channel variations and $\epsilon_{\mathrm{SER}}$ suffers a significant increase.

Considering that each frame contains $4 \%$ of pilot symbols and selecting $\alpha=0.4$, the effect of the preamble length is evaluated. Figure 4 plots the performance curves for preamble sizes of 8,16 and 80 BPSK symbols, respectively. For comparison purposes, we also include the result obtained using the supervised approach without including the decision rule. It can be seen that the preamble length does not influence the obtained SER, but it is an important parameter for reducing computational complexity ${ }^{2}$. Consequently, regardless of the preamble length required for the synchronization procedure, the decision rule will only consider the length of the preamble that will be included in the calculations of Eq. (10). In our evaluations we consider preambles with a length of 16 symbols. Additionally, Figure 4 also shows that the DA-Supervised approach achieves the same SER value as the supervised one.

In digital communication systems provided of feedback channels we have proposed to transmit pilot symbols only when a channel variation has been detected. For this reason, Fig. 4 (bottom) shows the percentage of pilot symbols that must be transmitted. This means that considering 16 preamble symbols, a SNR level higher than $6 \mathrm{~dB}$, and a Rayleigh-distributed channel, then a

\footnotetext{
2 The preamble length is a design parameter influenced mainly by the synchronization requirements. However, when implementing the proposed method to detect channel variations, one can decide to use the full preamble or just a portion of it.
} 
percentage of $25 \%$ of classical frames must be transmitted (including pilots), whereas the remaining $75 \%$ of frames contain only user data.

\subsection{Unsupervised Approaches}

In order to compare the performance achieved by the unsupervised approaches (i.e. JADE and BCEES) to that obtained when the decision criterion is also utilized (i.e. DA-JADE and DA-BCEES), we have considered that each frame contains 200 user data symbols. The preamble has 16 BPSK symbols, while the fourth-order cross-cumulants are estimated by sample averaging over 200 data symbols. Figure 5 shows both SER and algorithm utilization for the aforementioned approaches. The inclusion of the proposed decision criterion yields a considerable reduction in the computational complexity, whereas the obtained SER is not penalized since channel estimation is performed for a very small number of frames. Notice also that, as shown in Fig. 5, the difference - in terms of SER versus SNR - between JADE and DA-JADE with respect to BCEES and DA-BCEES increases with SNR. Consequently, although DABCEES offers a very low computational complexity, DA-JADE seems to be the best choice.

By comparing Fig. 5 and the curve corresponding to 16 preamble symbols in Fig. 4, we conclude that unsupervised approaches achieve the same performance as that of supervised ones without using pilot symbols and, for this reason, they are very attractive solutions for applications in which bandwidth is scarce and the additional complexity demanded by unsupervised techniques is affordable. 


\section{Performance Evaluation Based on Measured Indoor Channels}

In this section we describe the multiantenna testbed, the indoor scenario, and the measurement procedure followed to obtain the indoor wireless channel coefficients that are later on plugged in the simulations in order to evaluate the proposed approaches in real-world indoor channels. We carried out exactly the same simulations as those providing the results presented in Section 4, but replacing the synthetically-generated wireless channels by those estimated from a indoor testbed measurement campaign.

A testbed developed at the University of A Coruña [10] (see Fig. 6) was used to extract $4 \times 4$ channel matrices corresponding to a realistic indoor scenario in which the transmitter and the receiver were separated approximately $9 \mathrm{~m}$, whereas the antenna spacing was set to $7 \mathrm{~cm}$. Additionally, we have also developed a distributed multilayer software architecture, specifically designed to ease the interaction with the testbed $[8,10,11]$. More details about the testbed hardware description as well as similar measurement procedures to that included in this work can be found in [9] for a point-to-point MultipleInput Multiple-Output (MIMO) testbed and in [12] for a multiuser MIMO testbed.

\subsection{MIMO Testbed Description}

Both transmit and receive testbed nodes are equipped with a Quad DualBand front-end from Lyrtech, Inc. This Radio Frequency (RF) front-end can be equipped with up to eight antennas that are connected to four directconversion transceivers by means of an antenna switch. The front-end is based on Maxim MAX2829 chip (also found in front-ends like Ettus XCVR2450 or Sundance SMT911). It supports both up and down conversion operations from either a 2.4 to $2.5 \mathrm{GHz}$ band or a 4.9 to $5.875 \mathrm{GHz}$ band. The front-end 
also incorporates a programmable variable attenuator to control the transmit power value. The attenuation ranges from 0 to $31 \mathrm{~dB}$ in $1 \mathrm{~dB}$ steps, while the maximum transmit power declared by Lyrtech is $25 \mathrm{dBm}$ per transceiver.

The baseband hardware of all testbed nodes is based on Commercial OffThe-Shelf (COTS) components from Sundance Multiprocessor. More specifically, each transmit node is based on the SMT8036E kit, containing four Digital-to-Analog Converters (DACs) which generate Intermediate Frequency (IF) signals that feed the RF front-end only through the I branch (the Q branch is not used). Given that an IF signal is provided to a direct-conversion front-end, at its output we get the desired signal plus an undesired replica, which is suppressed at the receiver by shifting the RF carrier frequency and by adequate filtering later on in the digital domain.

Both transmit and receive nodes make use of real-time buffers which are used to store the signals to be sent to the DACs as well as the signals acquired by the Analog-to-Digital Converters (ADCs) (see Fig. 7). The utilization of such buffers allows for the transmission and acquisition of signals in real-time, while the signal generation and processing is carried out off-line. Additionally, both baseband hardware and RF front-ends of both nodes are synchronized in frequency by means of an external $40 \mathrm{MHz}$ reference oscillator.

\subsection{Measurement Procedure}

The block diagram of Fig. 7 shows the software and hardware elements utilized at both transmitter and receiver sides. In this case the testbed is used only to estimate the MIMO channel. For this purpose, we design a frame structure (see Fig. 8) consisting of a Pseudo-Noise (PN) sequence (119 symbols) for time and frequency synchronization; a silence (50 symbols) for estimating the noise variance at the receiver; and a long training sequence (4000 symbols 
per transmit antenna) for estimating the channel. The design of the frame structure pursues obtaining channel coefficients as accurate as possible, thus guaranteeing excellent time and frequency synchronization as well as estimates not significantly impacted by noise sources in the system. Therefore, the measurement procedure is driven by the quality of the channel estimations instead of pursuing spectral efficiency.

The signals employed to sound the channel are modulated (single carrier) and pulse-shape filtered using a squared root-raised cosine filter with $12 \%$ roll-off. The resulting signal bandwidth is $1.12 \mathrm{MHz}$, which leads -according to our tests- to a frequency-flat channel response.

With the aim of obtaining statistically rich channel realizations, and given that the Lyrtech RF front-end is frequency-agile, we measure at different RF carriers (frequency hopping) in the frequency interval ranging from $5219 \mathrm{MHz}$ to $5253 \mathrm{MHz}$ and from $5483 \mathrm{MHz}$ to $5703 \mathrm{MHz}$. Carrier spacing is $4 \mathrm{MHz}$ (greater than the signal bandwidth), which results in 65 different frequencies. Additionally, we repeat the whole measurement procedure for four different positions of the receiver, giving as a result 260 channel realizations. Note that we have these 260 realizations per each pair of transmit antennas for a given receiver position and therefore, taking into account the four receiver locations, a maximum number of 1040 channel realizations is available for the $(2 \times 1)$ Alamouti coding system.

In order to be able to plug the estimated channel coefficients in a simulation, all channel coefficients from each of the four sets of 65 channel matrices are normalized, giving as a result unit mean variance. 
5.3 Testbed Results

Figure 9 compares the probability density function (pdf) of the module of the channel coefficients acquired with the testbed to the pdf of syntheticallygenerated, spatially-white, Rayleigh-distributed wireless channels. Additionally, the histogram of both module and phase of the measured channel coefficients is also plotted. We can conclude from Fig. 9 that measured channels produce more attenuation in the received signals than for the case of Rayleighdistributed ones.

Figure 10 plots SER versus SNR for measured channels. Figure 11 plots SER and algorithm utilization percentage considering $4 \%$ of pilot symbols ( 8 pilot symbols). Compared to Fig. 3, it is apparent that the figure of merit defined in Eq. (11) is lower than that obtained for spatially-white Rayleighdistributed channels although the algorithm utilization is, however, similar.

We have also evaluated the performance with respect to the preamble length considering a threshold $\alpha=0.4$. Figure 12 compares the performance for a preamble length of 8,16 and 80 , and for the supervised approach without including the decision criterion. As for spatially-white Rayleigh-distributed channels (see Fig. 4), the preamble length influences only the computational complexity, but not SER. Again, the DA-Supervised algorithm achieves the same performance in terms of SER versus SNR as the supervised approach.

Finally, in Fig. 13 it can be seen that DA-JADE and DA-BCEES achieve the same performance in terms of SER versus SNR as JADE and BCEES, respectively, with a considerable reduction of the computational complexity. In this case, DA-BCEES does not present the degradation observed in the simulated channels and obtains the same performance as that offered by the supervised approach. 


\section{Conclusions}

The main idea proposed in this work is to use information obtained from time frame synchronization in order to track variations of the wireless channel. When variations are significant, the receiver estimates the channel matrix either using a supervised method or an unsupervised one. Otherwise, the decoding is performed using a previous channel estimate. The main advantage of the proposed method is that no additional operations are required relative to the conventional transmission system, and therefore, by selecting an adequate threshold value, it reaches the same performance as when the channel is estimated every frame.

We have shown that unsupervised approaches provide the same performance - in terms of SER versus SNR - as that offered by supervised ones, but without transmitting pilot symbols and, therefore, increasing the spectral efficiency. Consequently, they are attractive solutions for applications in which bandwidth is very scarce. The computational complexity of unsupervised methods is an important drawback for their utilization in energy-limited receivers and, for this reason, the inclusion of the proposed decision criterion represents a significant benefit. Hence, the decision-aided unsupervised approach arises as a promising method to avoid the transmission of training sequences, thus reducing power consumption in wireless communication devices.

Further work deals with testing the performance of the proposed decision rule in multicarrier systems where OFDM is used to transform multipath channels in several multiplicative channels similar to the considered in this paper. This research line also includes the evaluation taking into account the wireless standards specifications. 
Acknowledgements This work has been funded by Xunta de Galicia, Ministerio de Ciencia e Innovación of Spain, and FEDER funds of the European Union under grants with numbers 2012/287, TEC2010-19545-C04-01, and CSD2008-00010.

\section{References}

1. Alamouti, S.M.: A simple transmit diversity technique for wireless communications. IEEE Journal on Selected Areas in Communications 16, 1451-1458 (1998)

2. Beres, E., Adve, R.: Blind channel estimation for orthogonal STBC in MISO systems. In: Proc. of Global Telecommunications Conference (GLOBECOM 2004), vol. 4, pp. $2323-2328(2004)$

3. Cardoso, J.F., Souloumiac, A.: Blind beamforming for non-Gaussian signals. IEEE Proceedings-F 140(6), 362-370 (1993)

4. Castro, P.M., García-Naya, J. A. Dapena, A., Iglesia, D.: Channel estimation techniques for linear precoded systems: Supervised, unsupervised and hybrid approaches. Signal Processing, electronic publication (2011). DOI 10.1016/j.sigpro.2011.01.001

5. Comon, P., Jutten, C.: Handbook of Blind Source Separation, Independent Component Analysis and Applications. Academic Press (2010)

6. Dapena, A., Castro, P., García-Naya, J.A.: Hybrid supervised-unsupervised channel estimation scheme with dynamic transmission of pilots. Neural Processing Letters 33 $1-15(2011)$

7. Dapena, A., Pérez-Iglesias, H., Zarzoso, V.: Blind channel estimation based on maximizing the eigenvalue spread of cumulant matrices in (2x 1) Alamouti's coding schemes. Wireless Communications and Mobile Computing, electronic publication (2010). DOI $10.1002 /$ wcm.992

8. Fernández-Caramés, T.M., García-Naya, J.A., González-Lopez, M., Castedo, L.: MIMO testbed middleware for transmission automation. In: Proc. 50th International Symposium ELMAR, vol. 1, pp. 215-218. Zadar, Croatia (2008)

9. García-Naya, J.A., Fresnedo, O., Vázquez-Araújo, F.J., González-López, M., Castedo, L., Garcia-Frias, J.: Experimental evaluation of analog joint source-channel coding in indoor environments. In: Proc. IEEE International Conference on Communications (ICC 2011). Kyoto, Japan (2011)

10. García-Naya, J.A., González-López, M., Castedo, L.: Radio Communications, chap. A Distributed Multilayer Software Architecture for MIMO Testbeds. INTECH (2010) 
11. García-Naya, J.A., Perez-Iglesias, H.J., Fernández-Caramés, T.M., González-López, M., Castedo, L.: A distributed multilayer architecture enabling end-user access to MIMO testbeds. In: Proc. IEEE 19th International Symposium on Personal, Indoor and Mobile Radio Communications, pp. 1-5. Cannes, France (2008). DOI 10.1109/PIMRC.2008.4699692

12. González, O., Ramírez, D., Santamaría, I., García-Naya, J.A., Castedo, L.: Experimental validation of interference alignment techniques using a multiuser mimo testbed. In: Proc. International ITG Workshop on Smart Antennas (WSA 2011). Aachen, Germany (2011)

13. IEEE: IEEE standard for information technology- telecommunications and information exchange between systems- local and metropolitan area networks- specific requirements. part 11: Wireless LAN medium access control (MAC) and physical layer (PHY) specifications. amendment 5: Enhancements for higher throughput (2009). URL http://standards.ieee.org/getieee802/download/802.11n-2009.pdf

14. IEEE: IEEE standard for local and metropolitan area networks; part 16: Air interface for broadband wireless access systems, IEEE Std. 802.16-2009 (2009). URL http://standards.ieee.org/getieee802/download/802.16-2009.pdf

15. Love, D., Heath, R., Lau, V., Gesbert, D., Rao, B., Andrews, M.: An overview of limited feedback in wireless communication systems. IEEE Journal on Selected Areas in Communications 26(8), 1341-1365 (2008)

16. Minn, H., Bhargava, V., Letaief, K.: A robust timing and frequency synchronization for OFDM systems. IEEE Transactions on Wireless Communications 2(4), 822-839 (2003)

17. Morelli, M., Kuo, C.C., Pun, M.O.: Synchronization techniques for orthogonal frequency division multiple access (OFDMA): A tutorial review. Proceedings of the IEEE 95(7), 1394-1427 (2007). DOI 10.1109/JPROC.2007.897979

18. Pérez-Iglesias, H.J., García-Naya, J.A., Dapena, A., Castedo, L., Zarzoso, V.: Blind channel identification in alamouti coded systems: A comparative study of eigendecomposition methods in indoor transmissions at 2.4 GHz. European Transactions on Telecomunications 19(7), 751-759 (2008)

19. S., S., B., G.A., J., M.: Closed-form blind MIMO channel estimation for orthogonal space-time block codes. IEEE Transactions on Signal Processing 53(12), 4506-4516 (2005)

20. Stuber, G.L., Barry, J.R., Mclaughlin, S.W., Li, Y.G., Ingram, M.A., Pratt, T.G.: Broadband MIMO-OFDM wireless communications. Proceedings of the IEEE 92(2), 271-294 (2004) 
21. Tarokh, V., Jafarkhani, H., Calderbank, A.R.: Space-time block codes from orthogonal designs. IEEE Transactions on Information Theory 45(5), 1456-1467 (1999)

22. Vía J., S.I.: On the blind identifiability of orthogonal space-time block codes from second order statistics. IEEE Transactions on Information Theory 54(2), 709-722 (2008) 


\section{A Appendix}

In this appendix we will derive the maximum log-likelihood expression for channel coefficients used in the paper to estimation such channel coefficients from pilot sequences.

Under the assumptions A1 to A3, the expected log-likelihood of the observations is given, up to an irrelevant constant factor, by:

$$
L(\mathbf{h})=-\frac{1}{2 \sigma_{n}^{2} N} \sum_{k=0}^{N-1}\left\|\mathbf{x}_{k}-\mathbf{S}_{k} \mathbf{h}\right\|^{2}
$$

where $N$ is the number of samples of the observed signal. The gradient of Eq. (12) with respect to the unknown vector $\mathbf{h}$ yields the score function:

$$
\nabla L(\mathbf{h})=\frac{1}{\sigma_{n}^{2} N} \sum_{k=0}^{N-1} \mathbf{S}_{k}^{\mathrm{H}}\left(\mathbf{x}_{k}-\mathbf{S}_{k} \mathbf{h}\right)
$$

Setting the score to zero leads to the ML estimate:

$$
\hat{\mathbf{h}}_{\mathrm{ML}}=\frac{1}{2 N \hat{\sigma}_{s}^{2}} \sum_{k=0}^{N-1} \mathbf{S}_{k}^{\mathrm{H}} \mathbf{x}_{k}
$$

where $\hat{\sigma}_{s}^{2}=\frac{1}{2 N} \sum_{t=0}^{2 N-1}|s(t)|^{2}$ is a sample estimate of the source variance $\sigma_{s}^{2}$. To derive the expression of Eq. (14), we have exploited the orthogonality of the source symbol matrix $\mathbf{S}_{k}$ given by Eq. (3), which means in particular that

$$
\sum_{k=0}^{N-1} \mathbf{S}_{k}^{\mathrm{H}} \mathbf{S}_{k}=\sum_{k=0}^{N-1}\left(\left|s_{1}(k)\right|^{2}+\left|s_{2}(k)\right|^{2}\right) \mathbf{I}_{2}=\left(\sum_{t=0}^{2 N-1}|s(t)|^{2}\right) \mathbf{I}_{2}=2 N \hat{\sigma}_{s}^{2} \mathbf{I}_{2},
$$

where $\mathbf{I}_{2}$ is the $(2 \times 2)$ identity matrix. According to Eq. (14), the ML estimates of the channel coefficients can be expressed as follows:

$$
\begin{aligned}
& \hat{h}_{1, \mathrm{ML}}=\frac{1}{2 N \hat{\sigma}_{s}^{2}} \sum_{k=0}^{N-1}\left(s_{1}(k)^{*} x_{1}(k)-s_{2}(k) x_{2}(k)\right) \\
& \hat{h}_{2, \mathrm{ML}}=\frac{1}{2 N \hat{\sigma}_{s}^{2}} \sum_{k=0}^{N-1}\left(s_{2}(k)^{*} x_{1}(k)+s_{1}(k) x_{2}(k)\right) .
\end{aligned}
$$




\begin{tabular}{c|c}
\hline Preamble size & Base sequence \\
\hline 8 & $(1,-1)$ \\
16 & $(1,-1,-1,1)$ \\
80 & $(1,1,-1,-1,1,1,-1,-1,-1$ \\
& $1,1,1,1,1,-1,-1,-1,-1,-1)$ \\
\hline
\end{tabular}

Table 1 Base sequence used to generate the preambles of the first antenna.

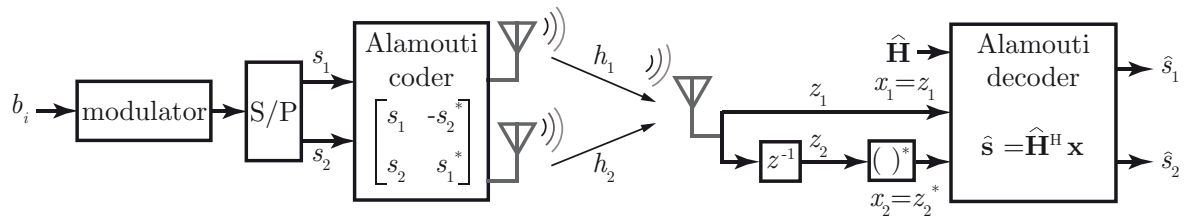

Fig. 1 Block diagram of the Alamouti OSTBC. 


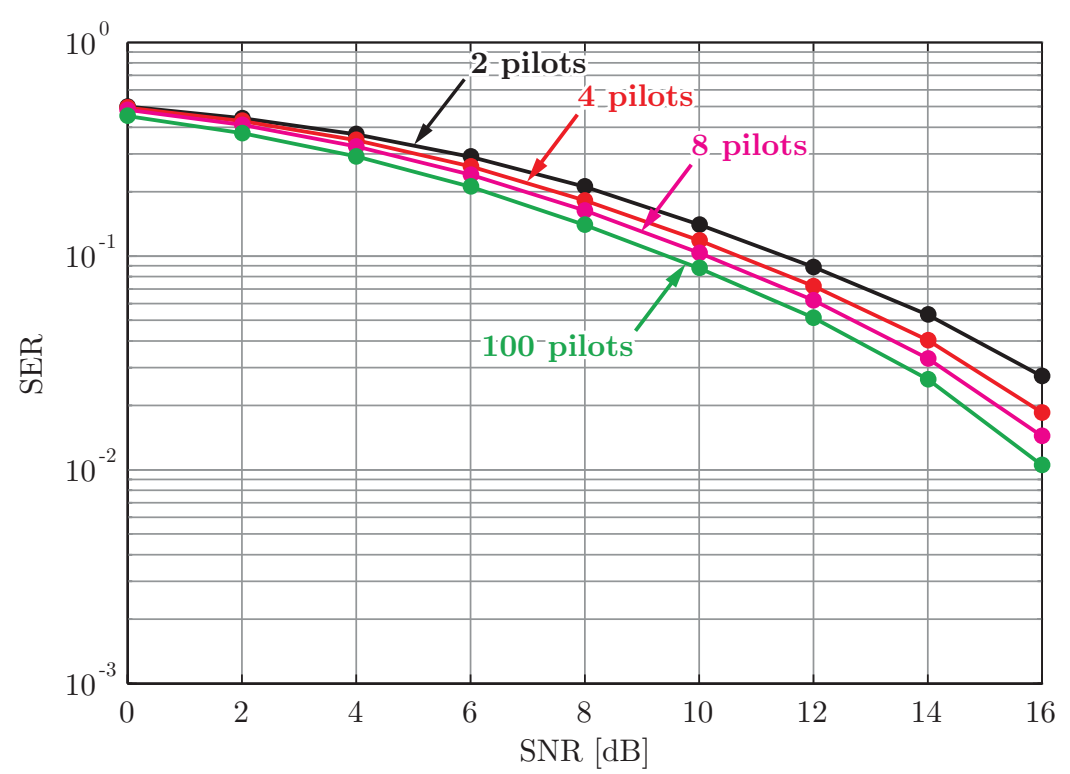

Fig. 2 SER versus SNR as a function of different percentages of pilot symbols used by the supervised approach for simulated channels. 


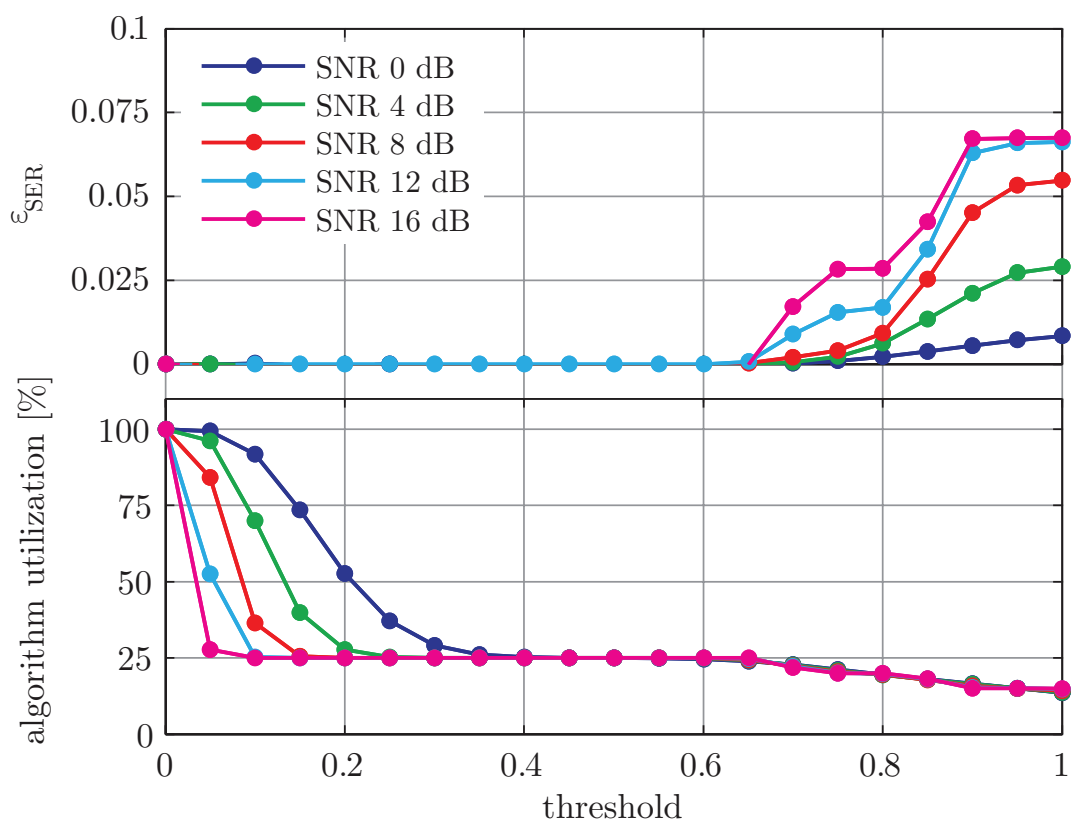

Fig. 3 SER and algorithm utilization for the supervised approach versus threshold $\alpha$ given several SNR values for simulated channels. 


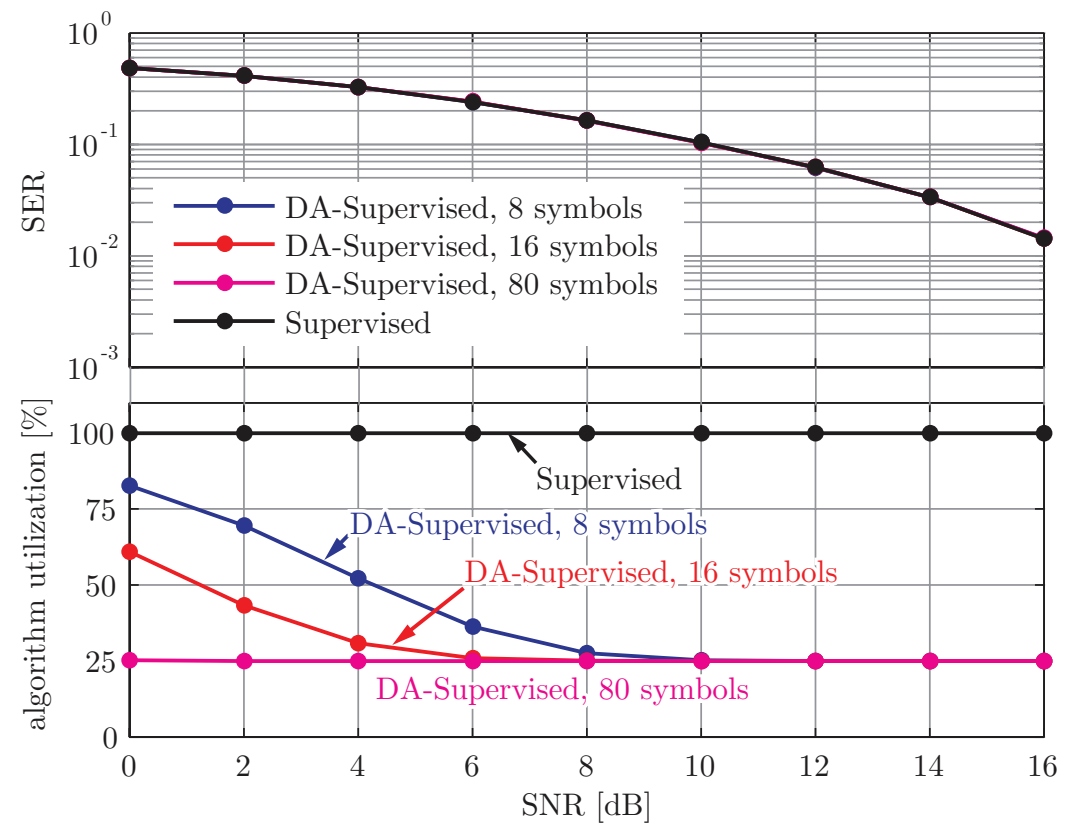

Fig. 4 SER and algorithm utilization for the supervised approach versus SNR depending on the preamble length for simulated channels. 


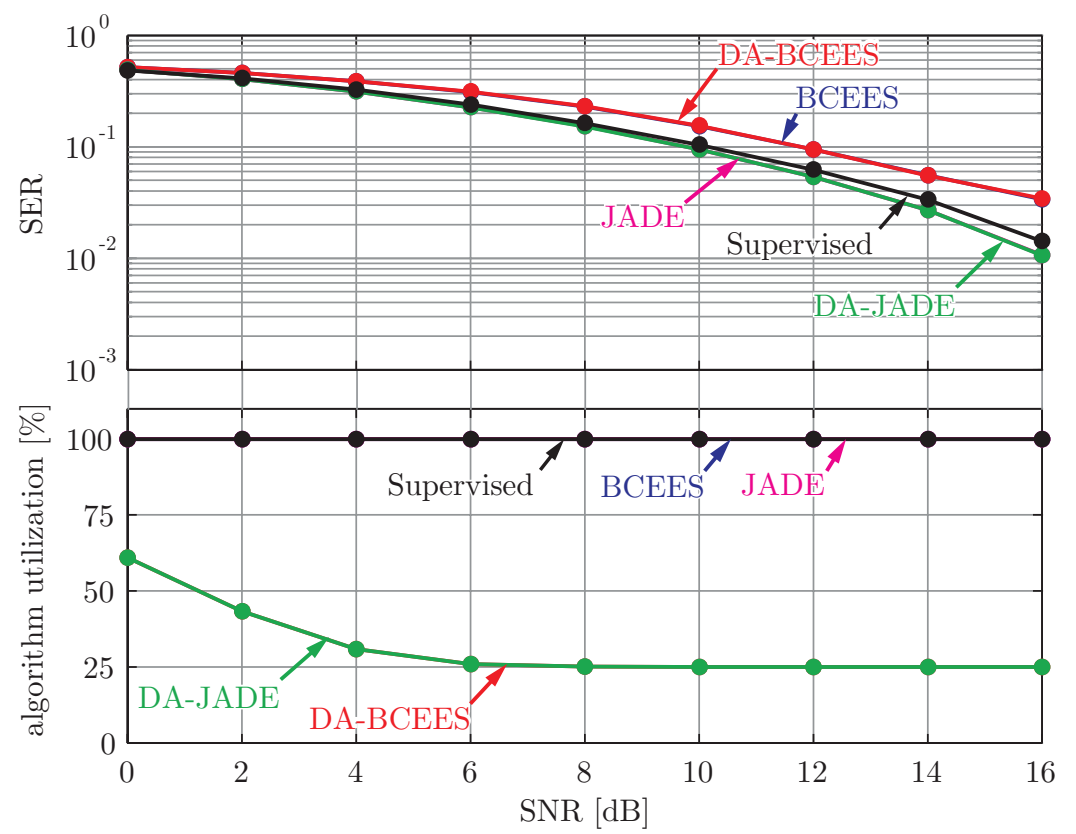

Fig. 5 SER and algorithm utilization versus SNR for simulated channels.

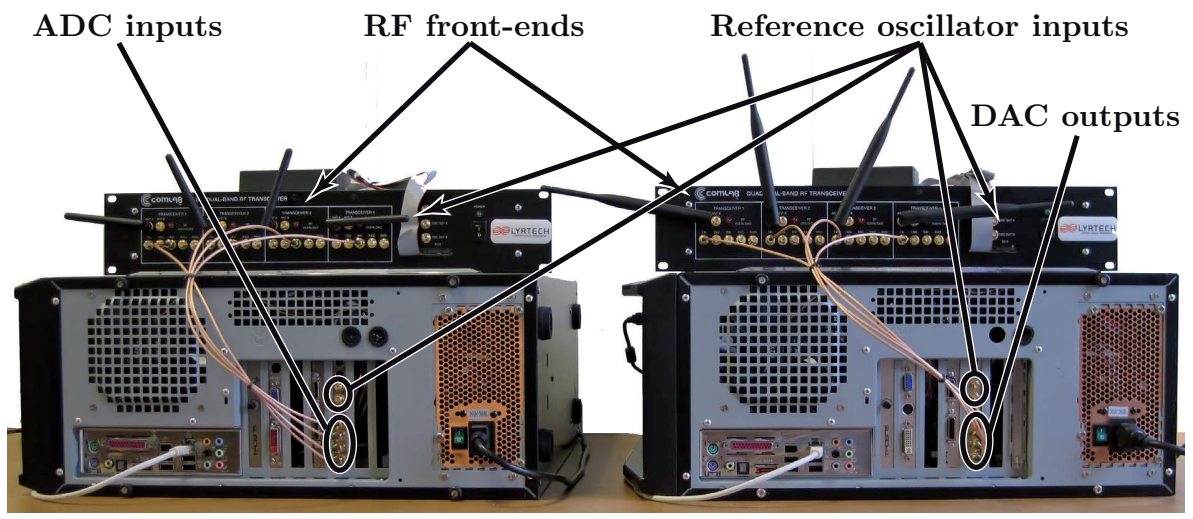

Fig. 6 Picture of the MIMO testbed. 

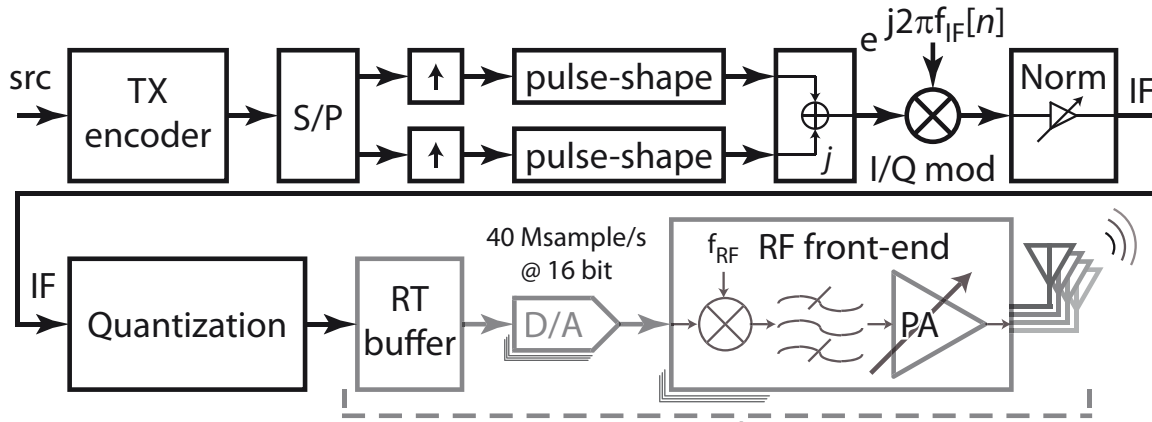

Real-time processing $\overline{-}-----\overline{\text { Real-time processing }} \overline{-}$
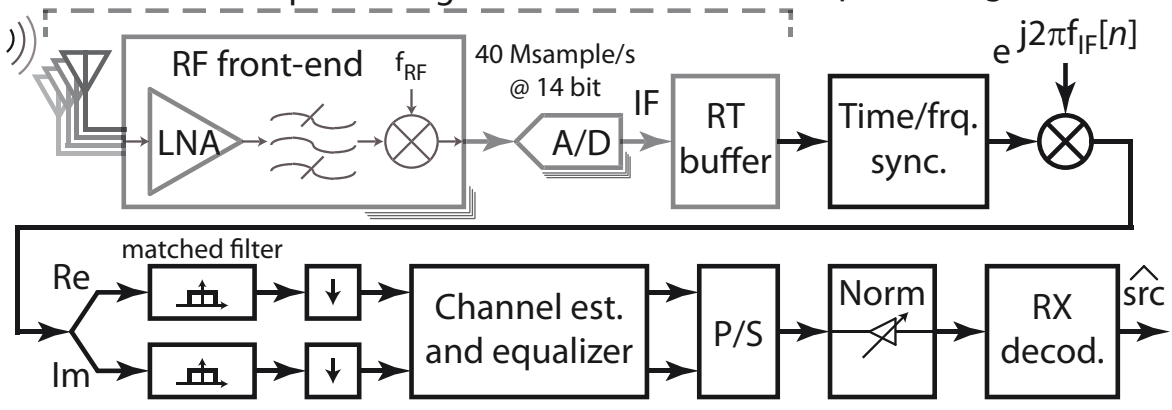

Fig. 7 Block diagram of hardware and software elements at both the transmitter and the receiver of the testbed.

\begin{tabular}{|c|c|c|}
\hline PN (preamble) & & training sequence (pilot symbols) \\
\hline 119 & 50 & 4000 (per TX antenna) \\
\hline
\end{tabular}

Fig. 8 Frame structure used to estimate the channel matrices. 

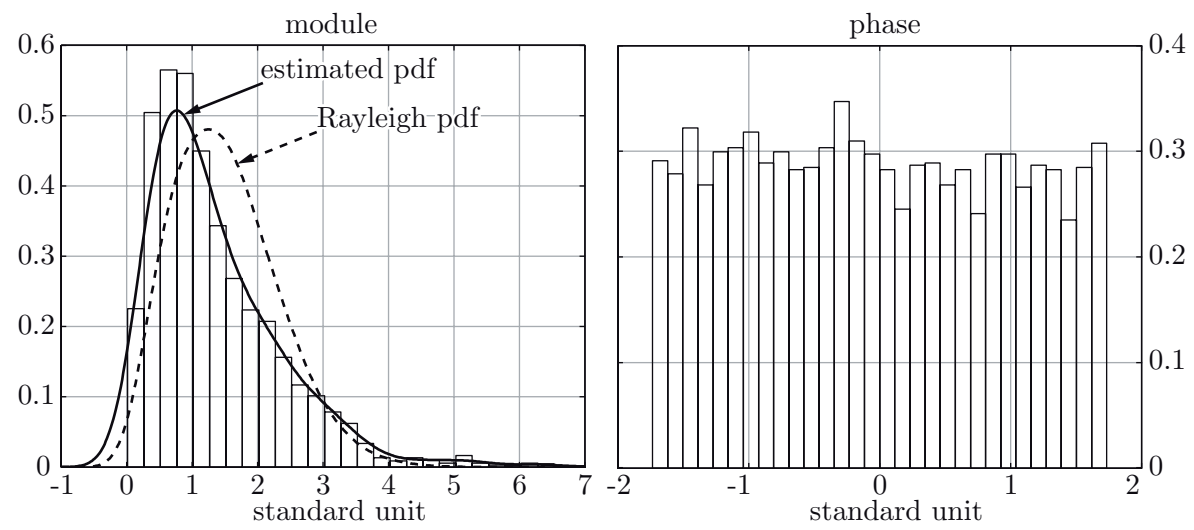

Fig. 9 Module (left-hand) and phase (right-hand) histograms for measured channels. Estimated pdf of the module for measured channels is compared to the Rayleigh pdf as well.

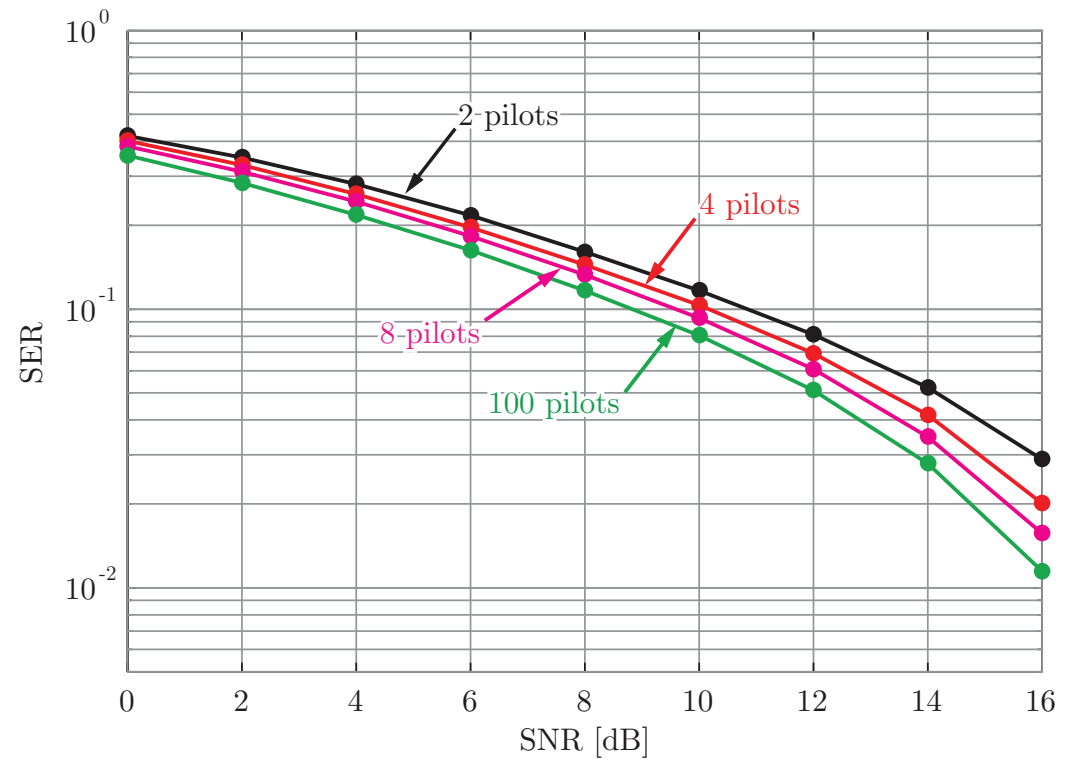

Fig. 10 SER versus SNR as a function of different percentages of pilot symbols used by the supervised approach for measured channels. 


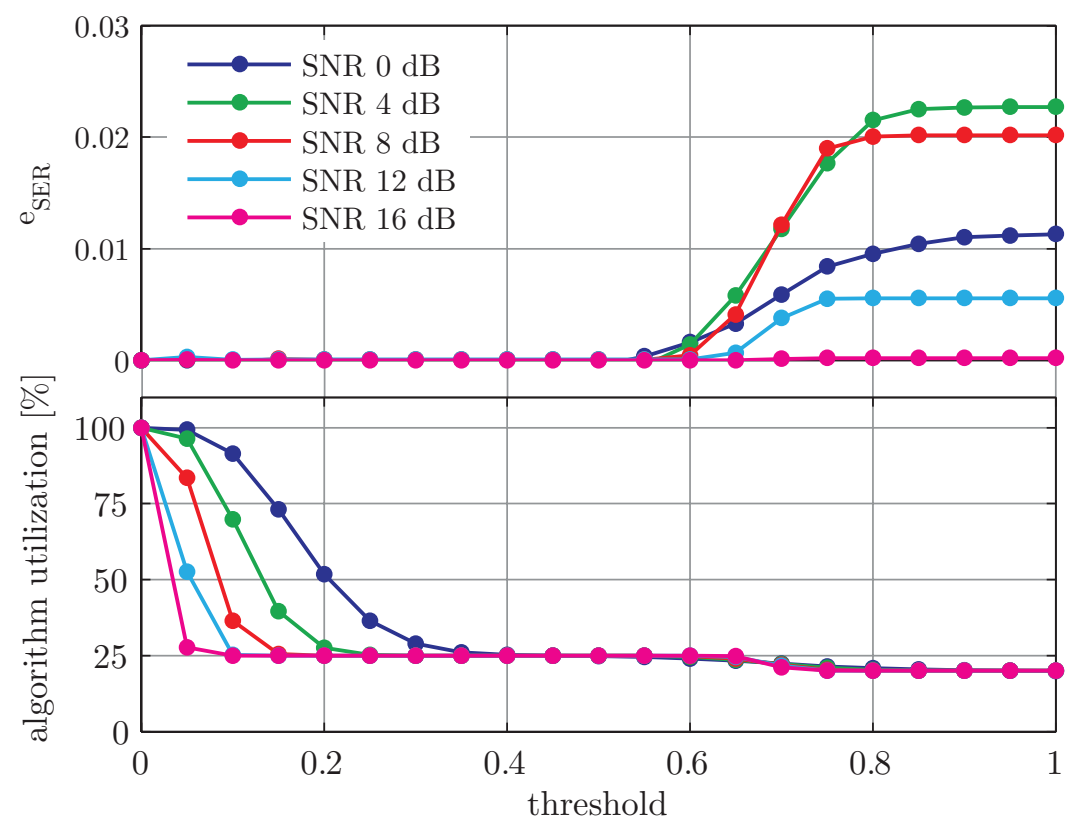

Fig. 11 SER and algorithm utilization for the unsupervised approach versus threshold $\alpha$ given several SNR values for measured channels. 


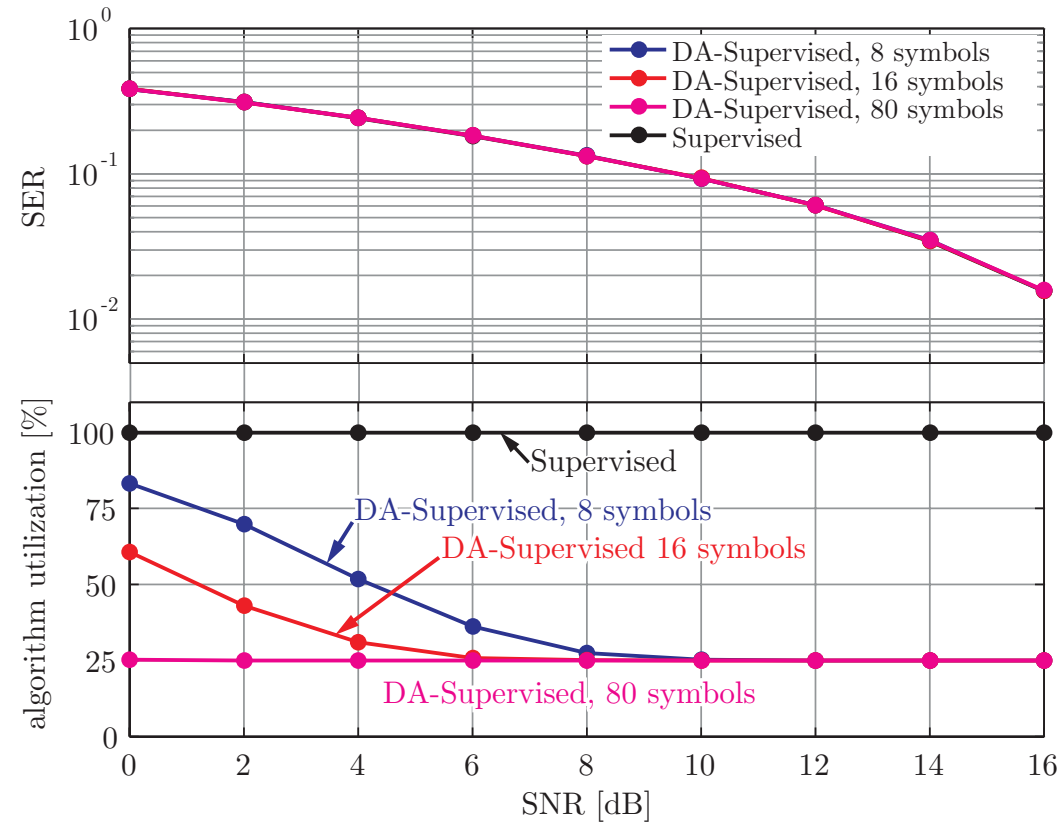

Fig. 12 SER and algorithm utilization for the supervised approach versus SNR depending on the preamble length for measured channels. 

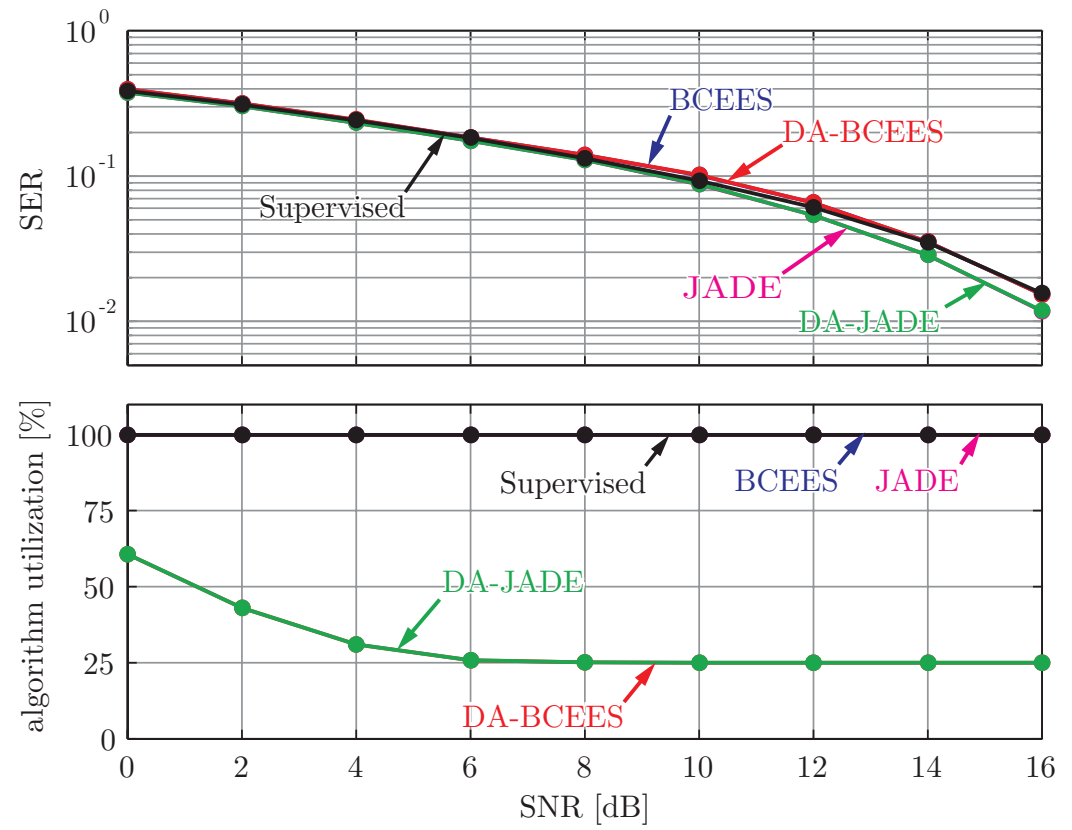

Fig. 13 SER and algorithm utilization versus SNR for measured channels. 